\title{
Which is Better: Stainless Steel or Titanium Alloy?
}

\author{
Sibel AKYOL ${ }^{1}$, Hakan BOZKUS ${ }^{2}$, Suzan ADIN CINAR ${ }^{3}$, Mehmet Murat $\mathrm{HANCI}^{4}$ \\ ${ }^{1}$ Istanbul University, Department of Physiology, Istanbul, Turkey \\ ${ }^{2}$ Istanbul Bilim University, Department of Neurosurgery, Istanbul, Turkey \\ ${ }^{3}$ Institute of Experimental Medicine, Istanbul, Turkey \\ ${ }^{4}$ Istanbul University, Department of Neurosurgery, Istanbul, Turkey
}

\section{ABSTRACT}

AIM: To investigate immunologic reactions after implantation of stainless steel (SS) alloy and titanium (Ti) alloy in a rat model. Macrophage and cytokine responses have been reported after in vivo and in vitro application of different biomaterials.

MATERIAL and METHODS: Wistar albino rats were used. After an exploration of the thoracolumbar paravertebral muscle tissue of the subjects, Group I underwent sham surgery, and Groups II and III underwent implantation of Ti alloy and SS alloy rods respectively. The CD4, CD8, CD25 (IL-2R) (lymphocyte and CD4 gate), CD4+CD8+ and CD4+CD25+Foxp3+ (Tregs), IL-4, IL-10, IL-6, IL-17A, TGF- $\beta$,TNF-a in the blood were analyzed.

RESULTS: CD4, CD25 (IL-2R), CD4+CD8+ and Tregs levels were lower in Group III compared to Group I (sham) and Group II. IL-6, IL-17A, TGF- $\beta$ and TNF-a levels in Group III showed a significant increase on all days in comparison with Group I and Group II. IL-4 and IL-10 levels were lower in Group III than those in Group II; and a significant decrease was observed in the IL-10 level. There was a reduction in IL-6 and IL-17A levels in Group II as opposed to Group I.

CONCLUSION: As opposed to SS alloy, Ti alloy suppresses the development of inflammation by inhibiting the pro-inflammatory response; strengthens the humoral immune system by intensifying the antibody-dependent immune response; triggers the development of immune tolerance by regulating the immune response; and activates the mechanism that prevents immune response-related damage from occurring.

KEYWORDS: Immune system, Implant, Stainless steel, Titanium

\section{INTRODUCTION}

$\mathrm{T}$

Temporary or permanent implants are used in stabilization surgeries of the human musculoskeletal system. Stainless steel (SS) alloy (SS 316L) and Titanium (Ti) alloy (Ti6Al4V), which are frequently used biomaterials for such implantations, are considered bio-inert materials.

One of the problems associated with the use of these biomaterials is that there is a possibility for the metal ions to separate from the implant in time and bind to biomolecules. Therefore, regardless of the corrosion of the implant, adverse tissue reactions, a promotion of infection, metal sensitivity, metal accumulation in the organs, and even possible cytotoxic effects have been reported $(6,7,9,17)$.
On the other hand, similar immuno-inflammatory tissue reactions have been detected when using SS alloy and $\mathrm{Ti}$ alloy. The presence of macrophages stained with CD68+ in the tissue surrounding the biomaterial was found to demonstrate that metal particles accumulate, and that the macrophages activated through the expression of HLA-DR induce the immune reaction of CD3+, CD4+, CD8+ T-lymphocytes by phagocytozing those metal particles $(12,23)$.

The aim of this study was to make a comparative assessment of the immune reactions to both SS alloy and Ti alloy implants in the postoperative period. 


\section{MATERIAL and METHODS}

\section{Experimental Animals and Metallic Implants}

The ethics committee approval for animal studies has been obtained from the Local Ethics Board for Animal Studies at the Istanbul University, as resolution Number 2013/26.

In the study, 39 Wistar albino male rats of 250-300 gram were randomly numbered and divided into 3 groups ( $n=13$ each). Group I was the sham surgery group, Group II was the Ti (Ti6Al4V) alloy rod group and Group III was the SS (SS 316L) alloy rod group.

Anesthetic agents ketamine $(50 \mathrm{mg} / \mathrm{kg})$ and xylazine (10 $\mathrm{mg} / \mathrm{kg}$ ) were administered intraperitoneally to each rat. Postanesthesia, the animals were placed on the operating table in the prone position. Prophylactic antibiotics were given intramuscularly before the surgery. At the thoracolumbar region, a $2 \mathrm{~cm}$ midline incision was made and the paravertebral region down to the lamina-facet junction was exposed by blunt dissection. No implant was used in Group I (the sham group). Rods were implanted on the lamina in Group II and III. The fascia and cutaneous tissue were closed with a 3/0 nonabsorbable suture.

Environmental conditions such as temperature $\left(25 \pm 2^{\circ} \mathrm{C}\right)$, light (12 hours light/dark), humidity (50-70\%) and ventilation of the room the animals were accommodated during the experiment were in compliance with relevant standards with automated lighting, an air conditioning device and ventilation system. Throughout the experiment, all rats were kept in polycarbonate cages, with a maximum of four animals per cage, and $250 \mathrm{~cm}^{2}$ of space per animal. All animals were fed with standard rat food ad-libitum.

The animals were monitored for physiological parameters and behavior throughout the experiment. The experiment was discontinued in case of a pathological change in physiological parameters or behavior of the animals. The subjects were also followed up for clinical symptoms including severe dehydration, hypothermia, hyperthermia, cachexia and anorexia. The experiment was terminated with cervical dislocation.

Considering that there might be changes in the immune parameters of female rats during their oestrus cycles, male Wistar albino rats were preferred for the experiment. Peripheral blood samples of approximately $1.5 \mathrm{cc}$ were drawn from all rats in all three groups before the surgery and on Day 7,14 and 28 after the surgery.

Surgical tools and rods to be used as implants were prepared in $1 \mathrm{~cm}$ pieces and sterilized before the surgery.

\section{Immunologic Analysis}

CD4, CD8, CD25 (IL-2R), CD4+CD8+ and CD4+CD25+Foxp3+ (Tregs) were measured using the flow cytometry method.

\section{Cytokine Analysis}

Interleukins (IL) such as IL-4, IL-10, IL-6, IL-17A, transforming growth factor- $\beta$ (TGF- $\beta$ ) and tumor necrosis factor- $\alpha$ (TNF- $a$ ) were measured using a multiple analyte platform (XMAP, Luminex).

\section{Statistics}

Descriptive statistics (mean, standard deviation) were performed to analyze the variables. The groups were compared with a one-way analysis of variance (ANOVA), and the Tukey's honest significant difference (HSD) test (for variables with homogenous variances) was used for the paired comparison of significant variables. The threshold for statistical significance was set at $p<0.05$.

\section{RESULTS}

The numerical values of the parameters analyzed in the study and their statistical significance have been presented in Figure 1 and Table I.

A schematic diagram has been presented in Figure 2 for a better understanding of the chain of immunologic responses that the biomaterials used in our study triggered.

The CD4+ cell response after SS alloy implantations (Group III) was found to be significantly reduced on Days 14 and 28 when compared to the control group (Group I) and Group II (Ti alloy rod). CD8+cells, on the other hand, which are an indicator of host-implant compatibility, did not present any change in the control or Group II, whereas they showed a tendency to increase in Group III (particularly on Day 14). CD4+ and CD8+ cells did not display any significant changes in Group II or Group III compared to Group I. However, while there was no significant change in the number of CD4+ and CD8+ cells in our study, a cascade might have been triggered because of a combination of signals resulting from an increased number of surface receptors and a stimulation of co-receptor activity, as well as cytokine release. This assumption follows the fact that the functions of $T$ cells and those of their subgroups, as well as cytokine release, were actively triggered in our study.

CD25 (IL-2R) levels in the SS alloy and Group II showed a significant and gradual decrease especially on Days 14 and 28. The decrease was more marked in Group III compared to Group II. CD25 (IL-2R, CD4+) levels in Group III decreased significantly when compared to Group I and Group II. There was no significant difference in CD25 (IL-2R, CD4+) levels between the latter two groups. T cell response was limited due to the decrease in the IL-2R level. Compared to Group I and Group II, CD4+CD8+ levels in Group III decreased significantly, especially on Days 14 and 28. Although there was also a decrease in Group II in CD4+CD8+ levels vis-à-vis Group I, it was not found to be significant. It is known that CD4+CD8+ T cells play role in the formation of a strong specific immune response to inflammation. The reduced CD4+CD8+ level observed in our study shows that the autoimmunity of the host is suppressed.

CD4+/CD25+Foxp3+(Tregs) levels decreased significantly on all days in Group III compared to the control group and Group II. In Group II, on the other hand, there was an increase in comparison with Group I. The increase was significant on Day 14, but was found to abate on Day 28.

We found a significant increase in the TNF-a level in Group III compared to the control and Group II. Group II, however, 
shows no significant difference in TNF-a level in comparison with Group I (Table I). This result suggests that there is a more marked pro-inflammatory response in Group III. It is known that inflammatory diseases and tissue damage might occur when Th1 functions cannot be balanced (10).

\section{DISCUSSION}

During the immune response following the implantation of a biomaterial, cytokines (pro- and anti-inflammatory mediators) are released as a result of the activation of leukocytes, and together with signaling protein molecules these cytokines coordinate a series of physiological processes such as wound healing, wound repair and immune reactions.

With the activation of macrophages, TNF and many IL cytokines (IL-1, IL-6, IL-8, IL-12, IL-15, IL-18, IL-23) are produced.
In addition to the aforementioned macrophage activation, lymphocytes perform a regulatory function via the Th1 cell and Th2 cell pathways. The Th1-cell response triggers cellmediated immune response with a release of cytokines such as IFN- $\gamma$ and IL-2, and the Th2-cell response activates B-lymphocytes by releasing cytokines like IL-4 and IL-10, all leading to a humoral immune response $(3,5)$. These cytokines are held responsible for infection, debris formation, bone resorption, osteoclast activity and bone remodeling after implantation.

CD4+ cells assume the role of a "conductor" orchestrating the response. Such a decrease in the number of CD4+ cells might cause a reduction in $\mathrm{MHC}$ class II molecules, complement receptors, the stimulation of $B$ lymphocytes, and the production of cytokines. In both intracellular and extracellular antigenic structures, these two cell groups control the immune

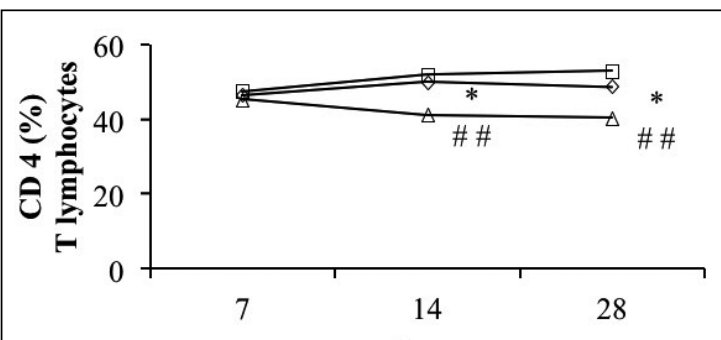

(A)

Days

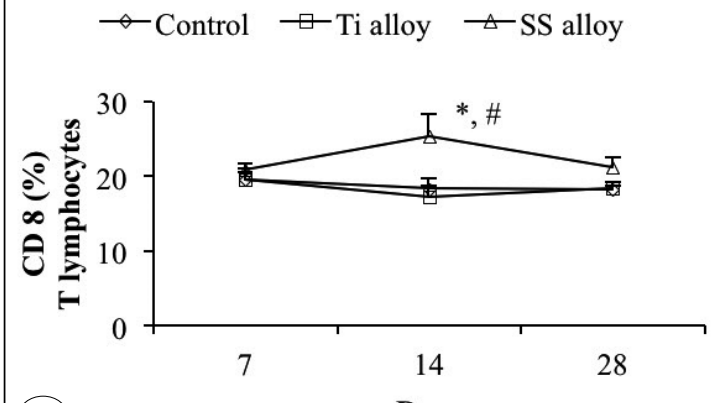

B

Days

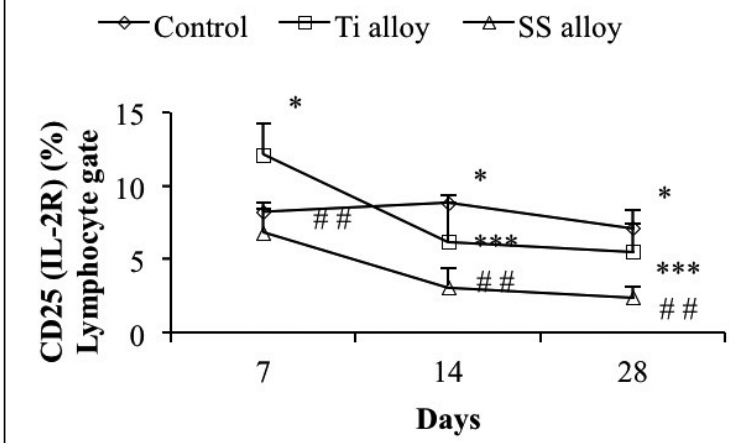

C $\neg$ Control $\square$ Ti alloy $\triangle$ SS alloy

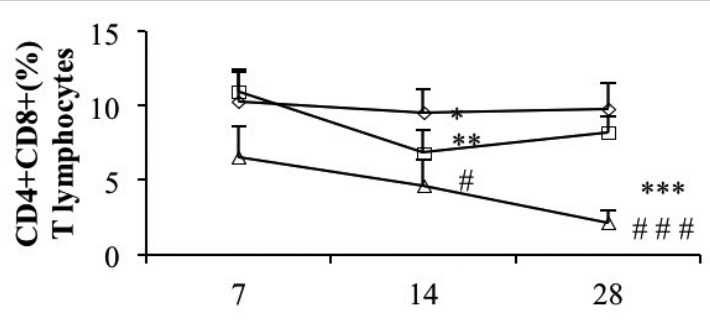

(D)

Days

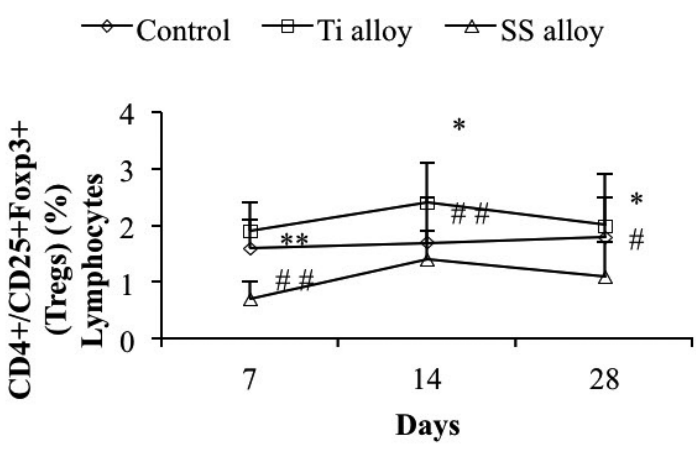

$\multimap$ Control $\square$ Ti alloy $\triangle$ SS alloy

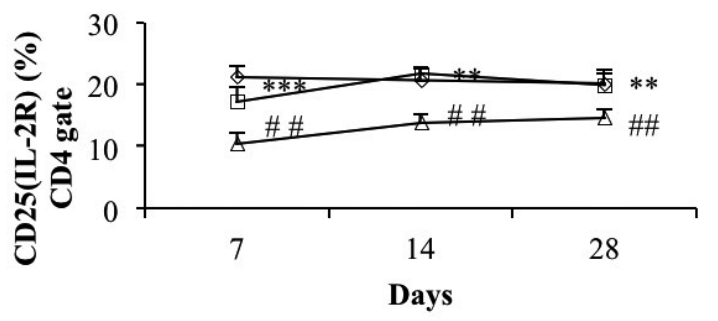

F $\rightarrow$ Control $\square$ Ti alloy $\triangle$ SS alloy

Figure 1: The comparison of cell levels (\%) in the control, Ti alloy and SS alloy groups between the days. A) CD4 T lymphocytes, B) CD8 T lymphocytes, C) CD25(IL-2R) lymphocytes, D) CD4+CD8+ T lymphocytes, E) CD4+/CD25+Foxp3+ (Tregs) lymphocyte gate, F) CD25 $(\mathrm{IL}-2 \mathrm{R}) \mathrm{CD} 4$ gate. ${ }^{*}\left(p\right.$ values, the groups compared to the control group); $p<0.05^{\star}, p<0.01^{\star *}, p<0.001^{\star \star *}$, \# ( $p$ values, the SS alloy group compared to the Ti alloy group); $p<0.05 \#, p<0.01 \# \#, p<0.001 \# \#$. 
response: $\mathrm{CD} 4+$ cells by producing cytokines and soluble mediators, and CD8+ cells by direct lysis (16).

IL-2 signals stimulate regulatory $T$ (Treg) cells through IL-2R. Tregs express a high level of IL-2Ra chains (CD25) and need IL-2 in order to continue developing. A decrease in the IL-2R level might increase affinity to autoimmunity by affecting $T$ cell homeostasis.

CD4+/CD25+Foxp3+ (Tregs) control destructive immune responses against pathogens by preventing the development of an immune response to self-antigens and harmless external antigens in the organism (22). Thus, self-tolerance develops, protecting the immune system from being overactive and eventually harming endogenous tissues $(15,24)$.

The increase in CD4+/CD25+Foxp3+(Tregs) in Group II following implantation controls the development of a destructive immune response by stimulating the peripheral regulatory mechanism. On the other hand, the decrease in the CD4+/ CD25+Foxp3+ (Tregs) level in Group III might trigger a cytotoxic and suppressive immune response, initiating pathological damage. CD4+/CD25+Foxp3+(Tregs) also suppress cytokine production (especially IL-2 production) by CD4+ and CD8+ responder $\mathrm{T}$ cells and their effector activities such as CD8+ T cell cytotoxicity.

Following metal implantation, metal ions have been reported to activate the release of various cytokines by coming into contact with peripheral T cells, thereby activating macrophages (15).

TNF- $a$ is a pro-inflammatory cytokine released by macrophages at an early stage and particularly responsible for systemic inflammatory symptoms, such as fever, and for the activation of other cytokines.

IL-6 is a pro-inflammatory cytokine released by $T$ cells and macrophages following infections and traumas. In contrast, there was a significant increase in Group II compared to Group I and Group III. The increase in IL-10 regulates immune effector functions, reduces the expression of inflammatory cytokines and suppresses the proliferation of $\mathrm{T}$ cytotoxic cells. Therefore, a possible interpretation is that the antiinflammatory cytokine response associated with IL-4 and IL10, manifests itself in a more effective way in Group II compared with Group III. The results from this study provide evidence of the possible function of IL-4 and IL-10 in the suppression of Group II particle-induced activation of macrophages. IL-4 may be a useful therapeutic agent in transplantation.

In contrast to Th1 and Th2 cells, the Th17 cell drives major neutrophils and other leukocyte subgroup cells to gather at the inflammation site via proinflammatory cytokines such as IL- 6 by causing the release of cytokines like IL-17 (IL-17A) and TNF- $a$ in inflammatory and autoimmune settings $(4,11,19)$. On the other hand, TGF- $\beta$ and IL-6, as pro-inflammatory cytokines, trigger the continuity of the immunologic response by enabling the differentiation of naive $\mathrm{T}$ (Th0) cells towards Th17 (8).

Th17 releases IL-17A pro-inflammatory cytokines. These cytokines cause destructive tissue damage in a delayedtype reaction by monocytes and neutrophils (to reach or

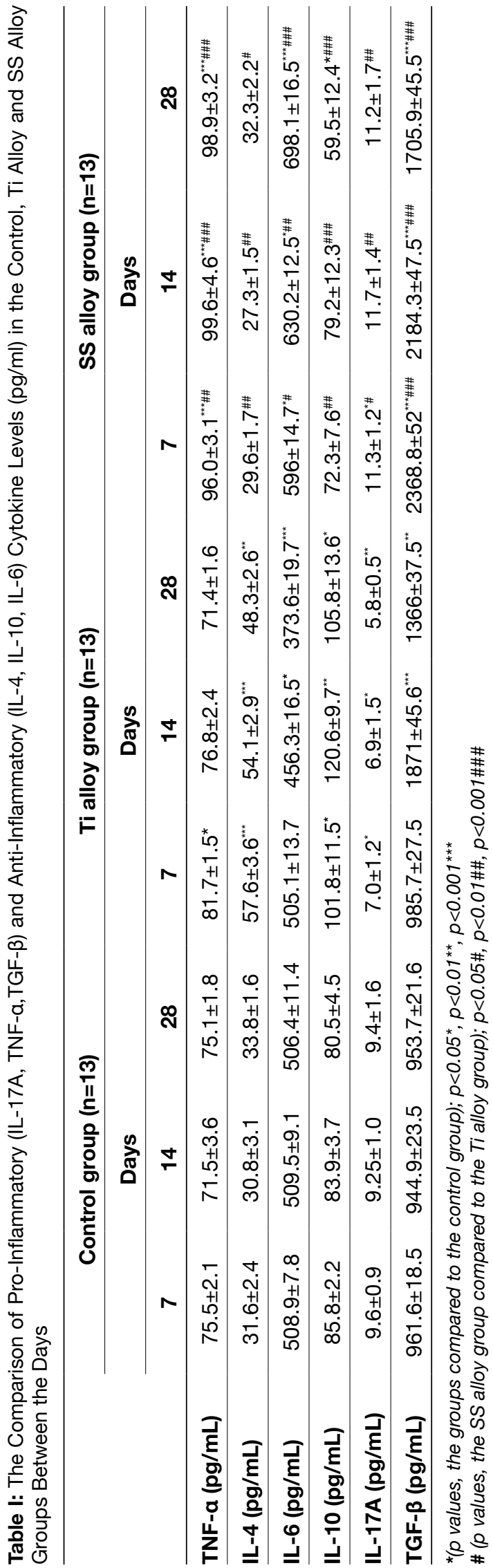


as they reach) the sites of inflammation. They play a role in the pathogenesis of autoimmune and acute transplant rejections. In case of extracellular damage, Th17 cells release high amounts of $\mathrm{IL}-17 \mathrm{~A}$ and play a significant role in the development of autoimmunity. IL-17A is a pleiotropic cytokine, which mediates tissue inflammation by inducing many pro-inflammatory cytokines such as IL-6 and TNF- $a$ and chemokines (18).

Ten percent of CD+4 T lymphocytes in the peripheral blood carry IL-2R (CD25) chains, and these cells called Tregs have been found to maintain tolerance against self-antigens by suppressing Th and B lymphocytes (25). Naive T cells that are exposed to TGF- $\beta$, an anti-inflammatory cytokine, induce Treg cells by expressing Foxp3 while IL-6 inhibits the induction of TGF- $\beta$-mediated Foxp3+ regulatory cells.

In our study, IL-17A levels in Group III are higher than those in Group I and Group II. Group II, on the other hand, showed a significant decrease compared with Group I. Therefore, it could be argued that the development of destructive tissue damage surrounding a metallic implant was more obvious in Group III vis-à-vis Group II.

$\mathrm{IL}-1 \beta$ and TNF- $\alpha$ secretions regulated by $\mathrm{IL}-17$ are suppressed by IL-4 and IL-10 cytokines (21). In our study, it could be argued that the effect of IL-17 on these type of cytokines could be reduced due to the increase of IL-4 and IL-10 levels in Group II compared to the SS alloy (Group III) and control groups.
TGF- $\beta$ is a protein that controls proliferation and cellular differentiation. Together with Foxp3+Treg, it regulates the immune system. In normal cells, TGF- $\beta$ stops proliferation by interrupting the cell cycle at the G1 stage. In our study, there was a significant increase in TGF- $\beta$ levels in the SS alloy and Group II compared to Group I. However, the increase in Group III was found to be more significant than that of Group II. Since CD25 (IL-2R) levels are lower in Group III than in Group II, the increased TGF- $\beta$ level causes a reduction in activity by suppressing IL-2 production by the T cells.

TGF- $\beta$ may act as a mediator of suppression as a membranebound form, although this is controversial. It may condition responder $\mathrm{T}$ cells to be sensitive to suppression and suppressive activity and might also contribute to the differentiation of other $T$ cells into Treg-like cells that results in infectious tolerance. While Treg cells cause homeostasis, they manifest their immunosuppressive effect in the release of suppressive cytokines such as IL-10 and TGF- $\beta$, their consumption of the IL-2 local concentration, which triggers apoptosis and stops the cell cycle at the cell-to-cell contact (13). Our findings and other findings suggest that Th17 cells and Treg cells balance the immune response together through a mutual antagonist interaction. On the other hand, according to our findings, it could be argued that the enabling of immune homeostasis in Group II is a result of a mutual antagonist interaction of Th17 cells with Treg cells by increasing tolerance in the immune system while producing an immune response that is not excessive and does not harm endogenous tissues.

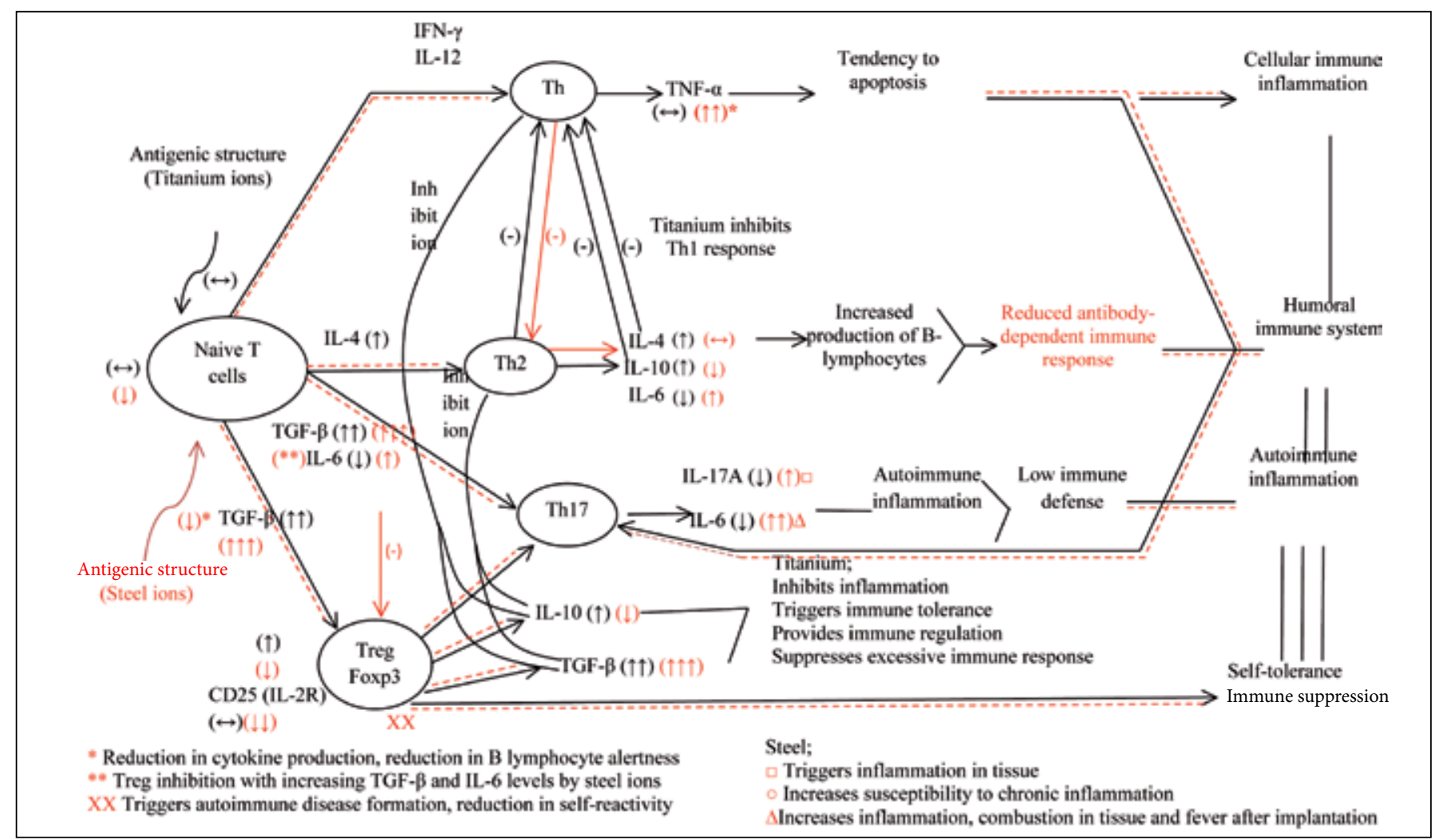

Figure 2: Schematic diagram shows immunologic pathway of titanium and stainless steel ions (black indicating the former, red indicating the latter). 
Some studies were shown that titanium implants produce less reaction than stainless steel plates (23). Other studies have reported infections when stainless steel and titanium implants were used in patients, independently from the material $(1,13,21)$.

In spinal surgery, when comparing Ti alloy and SS implants, only the SS implants cause late onset postoperative infections. Additionally, some degree of tissue loosening and corrosion has been observed (20). In our study, however, we showed a tendency to inflammation in the subjects that had stainless steel implants. At the same time, the anti-inflammatory parameters that ensure the immune defense system were lower for the stainless steel implants.

\section{- CONCLUSION}

Ti alloy implants suppress the development of inflammation by inhibiting the pro-inflammatory (Th1) response; strengthen the humoral immune system by accentuating the antibodydependent immune response (through a stimulation of $B$ lymphocytes and cytokines that enable the formation of antibodies); trigger the development of immune tolerance by regulating the immune response; and activate the mechanism that prevents the occurrence of immune response-related damage.

SS alloy implants increase the tendency for chronic inflammation and apoptosis; weaken the antibody-dependent immune response; cause fever after implantation (especially through the effect on IL-6 increase and prostaglandins); and trigger the mechanism that increases affinity to autoimmune diseases.

Compared to SS implants, Ti implants prevent possible harmful effects associated with the immune response by keeping immune activity within certain limits.

\section{REFERENCES}

1. Albrektsson T, Hansson HA: An ultrastructural characterization of the interface between bone and sputtered titanium or stainless steel surfaces. Biomaterials 7:201-205, 1986

2. Arens S, Schlegel U, Printzen G, Ziegler WJ, Perren SM, Hansis M: Influence of materials for fixation implants on local infection. An experimental study of steel versus titanium DCP in rabbits. J Bone Joint Surg Br 78:647-651,1996

3. Arora A, Song $Y$, Chun L, Huie P, Trindade M, Smith RL, Goodman S: The role of the TH1 and TH2 immune responses in loosening and osteolysis of cemented total hip replacements. J Biomed Mater Res Part A 64:693-697, 2003

4. Bettelli E, Carrier Y, Gao W, Korn T, Strom TB, Oukka M, Weiner HL, Kuchroo VK: Reciprocal developmental pathways for the generation of pathogenic effector $\mathrm{TH} 17$ and regulatory T cells. Nature 441:235-238, 2006

5. Borish LC, Steinke JW: Cytokines and chemokines. J Allergy Clin Immunol 111: 460-475, 2003

6. Daley B, Doherty AT, Fairman B, Case CB: Wear debris from hip or knee replacements causes chromosomal damage in human cells in tissue culture. J Bone Joint Surg $\mathrm{Br}$ 86-B:598606, 2004
7. Giertz SH, Fernandes BL, Fernandes CR, Franco CRC: Metallic ions in organs of rats injected with metallic particles of stainless steel 316L and Ti6Al4V alloy. Mat Res 13:35-39, 2010

8. Gregori S, Bacchetta R, Passerini L, Levings MK, Roncarolo $\mathrm{M}$ : Isolation, expansion, and characterization of human natural and adaptive regulatory T cells. Methods Mol Biol 380: 83-105,2007

9. Hanawa T: Metal ion release from metal implants. Mater Sci Eng C 24: 745-752, 2004

10. Im GI, Han JD: Suppressive effects of interleukin-4 and interleukin-10 on the production of proinflammatory cytokines induced by titanium-alloy particles. J Biomed Mater Res 58: 531-536, 2001

11. Jovanovic DV, Battista JAD, Martel-Pelletier J, Jolicoeur FC, He Y, Zhang M, Mineau F, Pelletier JP: IL-17 stimulates the production and expression of proinflammatory cytokines, IL- $\beta$ and TNF-a, by human macrophages. J Immunol 160:35133521,1998

12. Katou F, Andoh N, Motegi K, Nagura H: Immuno-inflammatory responses in the tissue adjacent to titanium miniplates used in the treatment of mandibular fractures. J Cranio-Maxillofac Surg 24:155-162,1996

13. Majetschak M, Bardenheuer M, Obertacke U: Immunoinflammatory tissue reaction to stainless-steel and titanium plates used for internal fixation of long bones. Biomaterials 24:247-254, 2003

14. Miyara M, Sakaguchi S: Natural regulatory T cells: Mechanisms of suppression. Trends Mol Med 13:108-116, 2007

15. Naka T, Nishimoto N, Kishimoto T: The paradigm of IL-6: From basic science to medicine. Arthritis Res 4:233-242, 2002

16. Palmer MT, Weaver CT: Autoimmunity; increasing suspects in the CD4+ T cell lineup. Nat Immunol 11(1):36-40, 2010

17. Rio JD, Beguiristain J, Duart J: Metal levels in corrosion of spinal implants. Eur Spine J 16(7):1055-1061, 2007

18. Sakaguchi S: Naturally arising CD4+ regulatory $T$ cells for immunologic self tolerence and negative control of immune responses. Ann Rev Immunol 22:531-562, 2004

19. Schmit-Weber CB, Akdis M, Akdis CA: TH17 cells in the big picture of immunology. J Allergy Clin Immunol 120:247-254, 2007

20. Soultanis KC, Pyrovolou N, Zahos K.A, Karaliotas GI, Lenti A, Liveris I, Babis GC, Soucacos PN: Late postoperative infection following spinal instrumentation: Stainless steel versus titanium implants. J Surg Orthop Adv Fall 17:193-199, 2008

21. Torgersen S, Moe G, Jonsson R: Immunocompetent cells adjacent to stainless steel and titanium miniplates and screws. Eur J Oral Sci 103:46-54, 1995

22. Vermes C, Kuzsner J, Bardos T, Peter T: Prospective analysis of human leukocyte functional tests reveals metal sensitivity in patients with hip implant. J Orthop Surg Res 8:1-7, 2013

23. Voggenreiter G, Leiting S, Brauer H, Leiting P, Majetschak $M$, Bardenheuer $\mathrm{M}$, Obertacke U: Immuno-inflammatory tissue reaction to stainless steel and titanium plates used for internal fixation of long bones. Biomaterials 24:247-254, 2003

24. Williams MA, Bevan MJ: Effector and memory CTL differentiation. Annu Rev Immunol 25:171-192, 2007

25. Wing K, Ekmark A, Karlsson H, Rudin A, Suri-Payer $E$ : Characterization of human CD25+CD4+ T cells in thymus, cord and adult blood. Immunology 106:190-199, 2002 